\title{
LOCAL ASYMPTOTIC SELF-SIMILARITY FOR HEAVY TAILED HARMONIZABLE FRACTIONAL LÉVY MOTIONS
}

\author{
Andreas Basse-O’Connor*io, ThorbJørn GrønbæK \\ AND MARK PODOLSKIJ
}

\begin{abstract}
In this work we characterize the local asymptotic self-similarity of harmonizable fractional Lévy motions in the heavy tailed case. The corresponding tangent process is shown to be the harmonizable fractional stable motion. In addition, we provide sufficient conditions for existence of harmonizable fractional Lévy motions.
\end{abstract}

Mathematics Subject Classification. 60G22, 60F05, 60E07.

Received January 4, 2018. Accepted June 15, 2021.

\section{INTRODUCTION}

The class of self-similar stochastic processes plays a key role in probability theory as they appear in some of the most fundamental limit theorems, see [9], and in modeling they are used in geophysics, hydrology, turbulence and economics, see [19] for numerous references. A self-similar process is by definition invariant in distribution under suitable time and space scaling, that is, a stochastic process $\left(X_{t}\right)_{t \in \mathbb{R}}$ is called self-similar with index $H \in \mathbb{R}$ if for all $c>0$ the two processes $\left(X_{c t}\right)_{t \in \mathbb{R}}$ and $\left(c^{H} X_{t}\right)_{t \in \mathbb{R}}$ are equal in finite dimensional distributions. The only self-similar centered Gaussian process with stationary increments is the fractional Brownian motion (up to scaling), which is a centered Gaussian process $\left(X_{t}\right)_{t \in \mathbb{R}}$ with $X_{0}=0$ a.s. and covariance function

$$
\operatorname{Cov}\left(X_{t}, X_{s}\right)=\frac{1}{2}\left(|t|^{2 H}+|s|^{2 H}-|s-t|^{2 H}\right) \quad \text { for all } s, t \in \mathbb{R},
$$

where $H \in(0,1)$. Only a very specific class of processes are exact self-similar, but a much larger class of processes behaves locally as a self-similar process - this is already seen within the class of Lévy processes.

A stochastic process $\left(X_{t}\right)_{t \in \mathbb{R}}$ is said to be locally asymptotically self-similar if there exists a number $H \in \mathbb{R}$ and a non-degenerate process $\left(T_{t}\right)_{t \in \mathbb{R}}$ such that

$$
\left(\frac{X_{\varepsilon t}}{\varepsilon^{H}}\right)_{t \in \mathbb{R}} \underset{\varepsilon \rightarrow 0_{+}}{\stackrel{d}{\longrightarrow}}\left(T_{t}\right)_{t \in \mathbb{R}}
$$

Keywords and phrases: Local asymptotic self-similarity; harmonizable processes; fractional processes; spectral representations.

Department of Mathematics, Aarhus University, Aarhus, Denmark.

* Corresponding author: basse@math.au.dk 
where $\stackrel{d}{\rightarrow}$ denotes convergence in finite dimensional distributions. The process $T=\left(T_{t}\right)_{t \in \mathbb{R}}$ is called the tangent process of $X$, and by (1.1), $T$ is necessarily self-similar. Local self-similarity means that at small time-scales the stochastic process $\left(X_{t}\right)_{t \in \mathbb{R}}$ is approximately self-similar and may be approximated by its tangent process. This property was introduced to provide a more flexible modeling framework compared to global self-similarity, $c f$. $[5,11]$. For applications, it has been used to study Internet traffic data, see [17], and for showing high frequency asymptotic results, see [1] or [2].

Moving average fractional Lévy motions: Starting from the moving average representation of the fractional Brownian motion, [10] has, among many others, studied fractional Lévy processes defined as

$$
X_{t}=\int_{-\infty}^{t}\left((t-s)_{+}^{\beta}-(-s)_{+}^{\beta}\right) \mathrm{d} L_{s}, \quad t \in \mathbb{R}
$$

where $\beta \in(0,1 / 2)$ and $\left(L_{t}\right)_{t \in \mathbb{R}}$ is a centered Lévy process with finite second moment. Throughout this paper $x_{+}:=\max \{x, 0\}$ and $x_{-}:=-\min \{x, 0\}$ denote the positive and negative parts of any number $x \in \mathbb{R}$.

In the following, we will call such processes for moving average fractional Lévy motions to distinguish them from their harmonizable counterpart. Under a regular variation assumption on the Lévy measure of $L$ near zero, Theorems 4.4 and 4.5 of [10] show that a moving average fractional Lévy motion is never self-similar, but it is locally asymptotically self-similar with tangent process the linear fractional stable motion, which is a process of the form (1.2) with $L$ being an $\alpha$-stable Lévy process. Slightly prior to [10], Proposition 4.1 of [4] proved a similar asymptotic result for a class of well-balanced moving averages.

Harmonizable fractional Lévy motions: Next we define the class of harmonizable fractional Lévy motions which includes the harmonizable fractional stable motion introduced in [6].

Definition 1.1. A stochastic process $\left(X_{t}\right)_{t \in \mathbb{R}}$ is called a harmonizable fractional Lévy motion with parameters $(\alpha, H) \in \mathbb{R}^{2}$ if

$$
X_{t}=\int_{\mathbb{R}} \frac{e^{i t s}-1}{i s}\left(a\left(s_{+}\right)^{-H-1 / \alpha+1}+b\left(s_{-}\right)^{-H-1 / \alpha+1}\right) \mathrm{d} L_{s}, \quad t \in \mathbb{R}
$$

where $a, b \in \mathbb{R}$ and $L$ is an isotropic complex-valued Lévy process.

Throughout the paper we define $0^{x}:=0$ for any $x \in \mathbb{R}$. The processes defined in Definition 1.1 are not always well-defined, but we will provide sufficient conditions for their existence. We refer to Section 3 for preliminaries on complex-valued processes and integrals. The (over) parametrization is chosen due to our forthcoming Assumption (A). In fact, under Assumption (A) below, the $H$ parameter in Definition 1.1 turns out to be exactly the number $H$ in the definition of local asymptotic self-similarity. From Theorem 4.1, below, it follows that harmonizable fractional Lévy motions have stationary increments and isotropic distributions. Furthermore, we give concrete conditions for existence of the harmonizable fractional Lévy motion on $(\alpha, H)$ and the Lévy measure of $L$.

In Proposition 3.1 of [3], local asymptotic self-similarity is studied for a slightly different class of harmonizable fractional motions under the assumption of finite second moment, that is, the Lévy measure $\nu$ of the Lévy process $L$ satisfies

$$
\int_{|x|>1}|x|^{2} \nu(\mathrm{d} x)<\infty
$$

Their result is the following:

Theorem 1.2 (Benassi, Cohen and Istas). Let $\left(X_{t}\right)_{t \in \mathbb{R}}$ denote a real harmonizable fractional Lévy motion as in Definition 2.3 of [3] satisfying the moment condition (1.4). Then the process $X$ is locally asymptotically 
self-similar with index $H$ and tangent process the fractional Brownian motion, that is,

$$
\left(\frac{X_{\varepsilon t}}{\varepsilon^{H}}\right)_{t \in \mathbb{R}} \underset{\varepsilon \rightarrow 0_{+}}{\stackrel{d}{\longrightarrow}}\left(B_{t}^{H}\right)_{t \in \mathbb{R}}
$$

where $\left(B_{t}^{H}\right)_{t \in \mathbb{R}}$ is a fractional Brownian motion with Hurst index $H$.

We note that Proposition 3.1 of [3] is stated under a stronger moment condition, however, for the statement in Theorem 1.2 above, only the second moment condition is used. The main aim of this work is to characterize the local asymptotic self-similarity of the harmonizable fractional Lévy motion when $L$ has heavy tails, violating the second moment condition (1.4). More precisely, we consider the case where the Lévy measure $\nu$ is regular varying in the following sense:

Assumption (A): Suppose that L is an isotropic complex-valued Lévy process without Gaussian component and let $\nu$ denote its Lévy measure. We assume that $\nu$ is absolutely continuous with respect to the two dimensional Lebesgue measure with a density $h: \mathbb{R}^{2} \rightarrow \mathbb{R}_{+}$satisfying

$$
\begin{aligned}
& h(x) \sim c_{0}|x|^{-2-\alpha} \quad \text { as }|x| \rightarrow \infty \quad \text { and } \\
& h(x) \leq C|x|^{-2-\alpha} \quad \text { for all } x \in \mathbb{R}^{2},
\end{aligned}
$$

where $c_{0}, C>0$.

The notation $\sim$ used in (1.5) means that the quotient between the left- and right-hand side of $\sim$ converges to 1 . When $a=b, \Re\left(X_{t}\right)$ is the real harmonizable fractional Lévy motion defined in Definition 2.3 of [3], and hence Theorem 1.2 applies when Assumption (A) holds with $\alpha>2$. The following theorem, which is the main result of this paper, characterizes the local asymptotic self-similarity of harmonizable fractional Lévy motions in the heavy-tailed case $\alpha<2$, and provides an additional existence result for them.

Theorem 1.3. Let $(\alpha, H) \in(0,2) \times(0,1)$ and suppose that Assumption $(A)$ is satisfied. Then the harmonizable fractional Lévy motion $X=\left(X_{t}\right)_{t \in \mathbb{R}}$, defined in (1.3), is well-defined, has stationary increments and isotropic distribution. Moreover, $X$ is locally asymptotically self-similar with index $H$ and tangent process the harmonizable fractional stable motion, i.e.

$$
\left(\frac{X_{\varepsilon t}}{\varepsilon^{H}}\right)_{t \in \mathbb{R}} \underset{\varepsilon \rightarrow 0_{+}}{\stackrel{d}{\longrightarrow}}\left(C_{t}\right)_{t \in \mathbb{R}}
$$

where the convergence is in finite dimensional distributions and $\left(C_{t}\right)_{t \in \mathbb{R}}$ is an isotropic harmonizable fractional stable motion with parameters $(\alpha, H)$, defined in equation (4.1) of [6].

We note that the tangent process in Theorem 1.3 differs from the tangent processes appearing in Theorem 1.2 and Theorem 4.5 of [10]. In Assumption (A) we assume that $h$ is regular varying at $\pm \infty$, whereas in the moving average case ([10], Thm. 4.50), $h$ is assumed to be regular varying at 0 . In particularly we observe that the smallscale behavior of $X$, is determined by the behavior of the Lévy measure of $L$ near zero in the moving average setting, whereas it is determined by Lévy measure near $\pm \infty$ in the harmonizable setting. The structure of the paper is as follows: Section 2 explains the role played by harmonizable processes within the class of stationary processes. Section 3 introduces complex random measures and their integration, whereas Section 4 concludes the paper with an existence criterion for harmonizable processes together with the proof of Theorem 1.3.

\section{BACKGROUND ON HARMONIZABLE PROCESSES}

Stationary processes form one of the main classes of stochastic processes. For stationary, centered Gaussian processes, it is well-known that every $L^{2}$-continuous process $\left(X_{t}\right)_{t \in \mathbb{R}}$ has a harmonizable representation of the 
form

$$
X_{t}=\int_{\mathbb{R}} e^{i t s} M(\mathrm{~d} s), \quad t \in \mathbb{R}
$$

for some complex-valued Gaussian random measure $M$ defined on $\mathbb{R}$. Furthermore, a rather large class of these processes has, in addition, a moving average representation, that is, a representation of the form

$$
X_{t}=\int_{\mathbb{R}} g(t-s) \mathrm{d} B_{s}, \quad t \in \mathbb{R}
$$

where $g$ is a deterministic function and $\left(B_{t}\right)_{t \in \mathbb{R}}$ is a two-sided real-valued Brownian motion. (Note that, the Brownian motion may be viewed as a shift-invariant Gaussian random measure.) Indeed, the class of Gaussian processes having a moving average representation corresponds exactly to those processes with absolute continuous spectral measure $\mu$. Recall that the spectral measure $\mu$ is given by $\mu(A)=\mathbb{E}\left[|M(A)|^{2}\right]$ for $A \in \mathcal{B}(\mathbb{R})$, where $M$ is given in (2.1). These classical results can be found in e.g. [7] or [20].

The only centered Gaussian self-similar process with stationary increments is the fractional Brownian motion $\left(B_{t}^{H}\right)_{t \in \mathbb{R}}$ with Hurst index $H \in(0,1)$, this process has the following two representations

$$
\begin{aligned}
& B_{t}^{H}=\int_{\mathbb{R}}\left((t-s)_{+}^{H-1 / 2}-(-s)_{+}^{H-1 / 2}\right) \mathrm{d} B_{s}, \quad \text { ("moving average representation") } \\
& B_{t}^{H}=\int_{\mathbb{R}} \frac{e^{i t s}-1}{i s}|s|^{-H-1 / 2+1} M(\mathrm{~d} s), \quad \text { ("harmonizable representation") }
\end{aligned}
$$

see Chapter 7.2 of [15] for further details. Hence, the fractional Gaussian noise $\left(B_{n}^{H}-B_{n-1}^{H}\right)_{n \in \mathbb{Z}}$ has both a harmonizable, (2.1), and a moving average, (2.2), representation. For comparison we will discuss the structure of stationary $\alpha$-stable processes with $\alpha \in(0,2)$ in the following.

In sharp contrast to the Gaussian situation the class of $\alpha$-stable stationary increments self-similar processes, $\alpha \in(0,2)$, is huge, and is far from being understood by now. However, two natural generalizations of the fractional Brownian motion to the $\alpha$-stable setting are proposed in [6] generalizing the fractional Brownian motion to $\alpha$-stable processes by replacing the driving Gaussian random measure with an $\alpha$-stable random measure in its moving average and harmonizable representations. This leads to the harmonizable fractional stable motion $\left(X_{t}\right)_{t \in \mathbb{R}}$, which is defined as

$$
X_{t}=\int_{\mathbb{R}} \frac{e^{i t s}-1}{i s}\left(a\left(s_{+}\right)^{-H-1 / \alpha+1}+b\left(s_{-}\right)^{-H-1 / \alpha+1}\right) \mathrm{d} L_{s}, \quad t \in \mathbb{R},
$$

where $\left(L_{t}\right)_{t \in \mathbb{R}}$ is a two-sided, complex-valued, $\alpha$-stable, isotropic Lévy process, and to the linear fractional stable motion $\left(X_{t}\right)_{t \in \mathbb{R}}$, which is defined as

$$
X_{t}=\int_{\mathbb{R}}\left(a\left((t-s)_{+}^{H-1 / \alpha}-(-s)_{+}^{H-1 / \alpha}\right)+b\left((t-s)_{-}^{H-1 / \alpha}-(-s)_{-}^{H-1 / \alpha}\right)\right) \mathrm{d} L_{s},
$$

where $\left(L_{t}\right)_{t \in \mathbb{R}}$ is a two-sided, real-valued, $\alpha$-stable, symmetric Lévy process. Notice that corresponding noise processes $\left(X_{n}-X_{n-1}\right)_{n \in \mathbb{Z}}$ for the linear and harmonizable fractional stable motions are moving average and harmonizable processes, respectively.

Indeed, the Gaussian assumption is crucial for the above equality between the harmonizable and moving average representations to hold, as it turns out that harmonizable fractional stable motion and linear fractional stable motion are quite different processes, $c f .[6]$ and [15]. The seminal paper [13] shows that every stationary $\alpha$ stable process has a unique decomposition into a (mixed) moving average component, a harmonizable component 
and a process of the "third kind", which does not admit moving average or harmonizable components. The class of mixed moving averages may be viewed as the class of processes having the least memory, whereas the class of harmonizable processes is the class having the largest degree of memory, and the processes of the third kind are in between. These facts come from ergodic consideration, see the introduction of [14] for more details, and are also illustrated by the fact that moving averages are always mixing and harmonizable processes are never ergodic nor mixing. Hence by studying moving averages and harmonizable processes, we are examining the two extremes of stationary $\alpha$-stable processes.

Thus, the comparison of results on local asymptotical self-similarity in the introduction between linear fractional stable motions and harmonizable fractional stable motions are, in fact, a comparison between $\alpha$-stable self-similar stationary increments processes with the least memory and with the most memory. This encircles the local asymptotical behavior of general $\alpha$-stable self-similar processes with stationary increments.

\section{PRELiminaries}

For $z \in \mathbb{C}$ we denote by $\Re(z)$ (resp. $\Im(z))$ the real (resp. complex) part of $z$, and $\tilde{z}=(\Re(z), \Im(z))$ is the corresponding vector in $\mathbb{R}^{2}$. In some cases we will identify $x=\left(x_{1}, x_{2}\right) \in \mathbb{R}^{2}$ with the complex number $x_{1}+i x_{2}$, e.g., if $z \in \mathbb{C}$ then $z x$ should be understood as a product of two complex numbers. By $\langle\cdot, \cdot\rangle$ we denote the usual inner product in $\mathbb{R}^{2}$ and $A^{T}$ denotes the transposed of a matrix $A$. All random variables and processes will be defined on a probability space $(\Omega, \mathcal{F}, \mathbb{P})$, and if $Z$ is a complex-valued random variable, then its characteristic function $\varphi_{Z}: \mathbb{R}^{2} \rightarrow \mathbb{C}$ is given by

$$
\varphi_{Z}(\theta)=\mathbb{E}\left[e^{i\langle\theta, \tilde{Z}\rangle}\right], \quad \theta \in \mathbb{R}^{2} .
$$

A complex-valued random variable $Z$ is said to have an isotropic distribution if for any orthogonal $2 \times 2$ matrix $A$, $A \tilde{Z}$ has the same distribution as $\tilde{Z}$. Similar, a stochastic process $\left(X_{t}\right)_{t \in \mathbb{R}}$ is said to have an isotropic distribution if for all $n \in \mathbb{N}, t_{1}, \ldots, t_{n} \in \mathbb{R}$ and $a_{1}, \ldots, a_{n} \in \mathbb{C}, \sum_{j=1}^{n} a_{j} X_{t_{j}}$ has an isotropic distribution. A process $\left(X_{t}\right)_{t \in \mathbb{R}}$ is said to have stationary increments if for all $h>0,\left(X_{t}-X_{0}\right)_{t \in \mathbb{R}}$ has the same finite dimensional distributions as $\left(X_{t+h}-X_{h}\right)_{t \in \mathbb{R}}$. Similarly, $L=\left(L_{t}\right)_{t \in \mathbb{R}}$ is a complex-valued Lévy process, if $L_{0}=0$ a.s., $L$ has càdlàg sample paths and stationary, independent increments, that is, $L_{t_{1}}-L_{t_{0}}, \ldots, L_{t_{n}}-L_{t_{n-1}}$ are independent for all $t_{0}<\cdots<t_{n}$ and $n \in \mathbb{N}$, and $L_{t+h}-L_{s+h}$ equals $L_{t}-L_{s}$ in distribution for all $h>0$ and $s<t$. We may identify $L$ with the two-dimensional Lévy process $\left(\tilde{L}_{t}\right)_{t \in \mathbb{R}}$, and hence there exists a characteristic triplet $(\gamma, \Sigma, \nu)$ where $\gamma \in \mathbb{R}^{2}, \Sigma$ is a symmetric non-negative definite $2 \times 2$ matrix, and $\nu$ is a Lévy measure on $\mathbb{R}^{2}$, that is, $\nu(\{0\})=0$ and $\int_{\mathbb{R}^{2}} \min \left\{1,|x|^{2}\right\} \nu(\mathrm{d} x)<\infty$ such that

$$
\varphi_{L_{1}}(\theta)=\exp \left(i\langle\theta, \gamma\rangle-\frac{1}{2}\langle\theta, \Sigma \theta\rangle+\int_{\mathbb{R}^{2}}\left\{e^{i\langle x, \theta\rangle}-1-i\langle x, \theta\rangle 1_{\{|x| \leq 1\}}\right\} \nu(\mathrm{d} x)\right)
$$

for all $\theta \in \mathbb{R}^{2}, c f$. Chapter 2 of [16]. In the case $\Sigma=0$ we will say that $L$ has no Gaussian component. Process $L$ is said to be symmetric if $-L_{1}$ equals $L_{1}$ in distribution, and in this case, we have $\gamma=0$ and $\nu$ is a symmetric measure. Any complex-valued Lévy process $L$ induces an independently scattered infinitely divisible random measure $M$ on $\mathbb{R}$ by setting $M((a, b])=L_{b}-L_{a}$ for all $-\infty<a<b<\infty$. The integral of a deterministic measurable function $f: \mathbb{R} \rightarrow \mathbb{C}$ with respect $L$ is defined through approximation of $f$ with simple functions, whenever the integral $\int f \mathrm{~d} L$ exists as a complex-valued random variable. The paper [18] provides necessary and sufficient conditions for the existence of the stochastic integral, however, the conditions from [8] (a multivariate extension of [12]) seem easier to verify in our setting:

Theorem 3.1 (Prop. 5.10 and Cor. 5.11 of [8]). Let $\left(L_{t}\right)_{t \in \mathbb{R}}$ denote a symmetric complex-valued Lévy process with Lévy measure $\nu$ and no Gaussian component. Moreover, let $f: \mathbb{R} \rightarrow \mathbb{C}$ be a measurable function of the 
form $f=f_{1}+i f_{2}$. Then $f$ is L-integrable if and only if the following condition hold true

$$
\int_{\mathbb{R}} \int_{\mathbb{R}^{2}} \min \left(1,|f(s) x|^{2}\right) \nu(\mathrm{d} x) \mathrm{d} s<\infty
$$

In the affirmative case, the characteristic function of $\int f \mathrm{~d} L$ is given by

$$
\begin{aligned}
& \varphi_{\int f \mathrm{~d} L}(\theta) \\
& \quad=\exp \left(\int_{\mathbb{R}} K\left(\theta_{1} f_{1}(s)+\theta_{2} f_{2}(s), \theta_{2} f_{1}(s)-\theta_{1} f_{2}(s)\right) \mathrm{d} s\right)
\end{aligned}
$$

for all $\theta=\left(\theta_{1}, \theta_{2}\right) \in \mathbb{R}^{2}$, where

$$
K(y)=\int_{\mathbb{R}^{2}}(\cos (\langle y, x\rangle)-1) \nu(\mathrm{d} x), \quad y \in \mathbb{R}^{2} .
$$

Remark 3.2. Let $L$ be a Lévy process as in Theorem 3.1 and assume, in addition, that $L$ is isotropic. Then $K$ from (3.2) is of the form $K(y)=\Psi(|y|)$ for some function $\Psi: \mathbb{R} \rightarrow \mathbb{R}$, and for all $L$-integrable functions $f: \mathbb{R} \rightarrow \mathbb{C}$ and all $\theta \in \mathbb{R}^{2}$ we have

$$
\log \varphi \int f \mathrm{~d} L(\theta)=\int_{\mathbb{R}} \Psi(|\theta||f(s)|) \mathrm{d} s
$$

Indeed, the representation of $K$ follows from the isotropic assumption on $L$. Moreover, (3.3) follows by (3.1) and

$$
\left|\left(\theta_{1} f_{1}(s)+\theta_{2} f_{2}(s), \theta_{2} f_{1}(s)-\theta_{1} f_{2}(s)\right)\right|=|f(s)||\theta|
$$

\section{Existence AND PROPERTIES OF HARMONIZABle FRACTIONAL LÉVy MOTIONS}

Since the class of harmonizable fractional Lévy motions is an important and flexible class of infinitely divisible processes, we give a general existence criterion for them in the following (going beyond Assumption (A)).

Theorem 4.1. Let $L=\left(L_{t}\right)_{t \in \mathbb{R}}$ be a complex-valued isotropic Lévy process with Lévy measure $\nu$ and no Gaussian component. The harmonizable fractional Lévy motion $X=\left(X_{t}\right)_{t \in \mathbb{R}}$, defined in Definition 1.1, with parameters $(\alpha, H) \in(0,2) \times(0,1)$ exists if the following $(a)-(c)$ are satisfied:

(a) $\int_{|x|>1}|x|^{\frac{1}{H+1 / \alpha}} \nu(\mathrm{d} x)<\infty$,

(b) $\int_{|x| \leq 1}|x|^{\frac{1}{H+1 / \alpha-1}} \nu(\mathrm{d} x)<\infty \quad$ when $H+1 / \alpha>3 / 2$,

(c) $\int_{|x| \leq 1}|x|^{2} \log \left(|x|^{-1}\right) \nu(\mathrm{d} x)<\infty \quad$ when $H+1 / \alpha=3 / 2$.

Furthermore, if $X$ exists, then it has stationary increments, isotropic distribution and

$$
\log \varphi_{X_{t}}(\theta)=\int_{\mathbb{R}} K\left(2 \sin (t s / 2)\left\{a\left(s_{+}\right)^{-H-1 / \alpha}+b\left(s_{-}\right)^{-H-1 / \alpha}\right\} \theta\right) \mathrm{d} s
$$

for all $t \in \mathbb{R}$ and $\theta \in \mathbb{R}^{2}$, where $K$ is given by (3.2).

For two functions $g_{1}$ and $g_{2}$, the notation $g_{1}(s)=O\left(g_{2}(s)\right)$ as $s \rightarrow 0$ means that $\limsup _{s \rightarrow 0}\left|g_{1}(s) / g_{2}(s)\right|<\infty$, and a similar notation is used when $|s| \rightarrow \infty$. To prove Theorem 4.1 we will first show the following lemma. 
Lemma 4.2. Let $\nu$ be a Lévy measure on $\mathbb{R}$ and let $f: \mathbb{R} \rightarrow \mathbb{R}_{+}$be a measurable function, bounded on $[-1,1]^{c}$, and satisfying

$$
f(s)=O\left(|s|^{\beta}\right) \text { as } s \rightarrow 0, \quad \text { and } \quad f(s)=O\left(|s|^{-\gamma}\right) \text { as }|s| \rightarrow \infty,
$$

for some $\beta \in \mathbb{R}$ and $\gamma>0$. Then

$$
\int_{\mathbb{R}} \int_{\mathbb{R}} \min \left(1,|x f(s)|^{2}\right) \nu(\mathrm{d} x) \mathrm{d} s<\infty
$$

if the conditions (a)-(c) hold:

(a) $\gamma>1 / 2$ and

$$
\int_{|x|>1}|x|^{\frac{1}{\gamma}} \nu(\mathrm{d} x)<\infty .
$$

(b) If $\beta<-1 / 2$ then

$$
\int_{|x| \leq 1}|x|^{-\frac{1}{\beta}} \nu(\mathrm{d} x)<\infty .
$$

(c) If $\beta=-1 / 2$ then

$$
\int_{|x| \leq 1} x^{2} \log \left(|x|^{-1}\right) \nu(\mathrm{d} x)<\infty
$$

Proof of Lemma 4.2. Assume that conditions (a)-(c) hold. By observing that the integral (4.3) is increasing in $f$, it suffices to study the integral (4.3) for a function $g: \mathbb{R} \rightarrow \mathbb{R}$ of the form $g(s)=\mathbb{1}_{[-1,1]}(s)|s|^{\beta}+\mathbb{1}_{[-1,1]^{c}}(s)|s|^{-\gamma}$, that is, to show

$$
\int_{\mathbb{R}} \int_{\mathbb{R}} \min \left(1,|x g(s)|^{2}\right) \mathrm{d} s \nu(\mathrm{d} x)<\infty .
$$

We divide the integration region $\mathbb{R} \times \mathbb{R}$ into the following four areas:

$$
\begin{aligned}
& A_{11}=\{(s, x) \in \mathbb{R} \times \mathbb{R}:|s| \leq 1,|x| \leq 1\}, \\
& A_{12}=\{(s, x) \in \mathbb{R} \times \mathbb{R}:|s| \leq 1,|x|>1\}, \\
& A_{21}=\{(s, x) \in \mathbb{R} \times \mathbb{R}:|s|>1,|x| \leq 1\}, \\
& A_{22}=\{(s, x) \in \mathbb{R} \times \mathbb{R}:|s|>1,|x|>1\} .
\end{aligned}
$$

We first consider $A_{22}$ and let $x \in[-1,1]^{c}$ be given. We write the inner integral in (4.5) as

$$
\int_{\{|s|>1\} \cap\left\{|s|>|x|^{1 / \gamma}\right\}}|x|^{2}|s|^{-2 \gamma} \mathrm{d} s+\int_{\{|s|>1\} \cap\left\{|s| \leq|x|^{1 / \gamma}\right\}} 1 \mathrm{~d} s=\frac{4 \gamma}{2 \gamma-1}|x|^{1 / \gamma}-2,
$$

where we have used that $\gamma>1 / 2(c f$. (a)). Inserting (4.6) we obtain

$$
\int_{A_{22}} \min \left(1,|x g(s)|^{2}\right) \nu(\mathrm{d} x) \mathrm{d} s=\int_{|x|>1}\left[\frac{4 \gamma}{2 \gamma-1}|x|^{1 / \gamma}-2\right] \nu(\mathrm{d} x) .
$$


Since $\nu(x \in \mathbb{R}:|x|>1)<\infty$, the last integral in (4.7) is finite if and only if

$$
\int_{|x|>1}|x|^{1 / \gamma} \nu(\mathrm{d} x)<\infty
$$

which we have assumed in (4.4). For $A_{11}$, let $x \in[-1,1] \backslash\{0\}$ be given. For $\beta \leq-1 / 2$ the inner integral in (4.5) can be written as

$$
\begin{array}{r}
\int_{\{|s| \leq 1\} \cap\left\{|s|^{\beta} \leq|x|^{-1}\right\}}|x g(s)|^{2} \mathrm{~d} s+\int_{\{|s| \leq 1\} \cap\left\{\left.|s|\right|^{\beta}>|x|^{-1}\right\}} \mathrm{d} s \\
=|x|^{2} \int_{1 \geq|s| \geq|x|^{-1 / \beta}}|s|^{2 \beta} \mathrm{d} s+\int_{\{|s| \leq 1\} \cap\left\{|s| \leq|x|^{-1 / \beta}\right\}} \mathrm{d} s \\
= \begin{cases}\frac{2 x^{2}}{2 \beta+1}\left(1-|x|^{-\frac{2 \beta+1}{\beta}}\right)+2|x|^{-1 / \beta} & \text { if } \beta<-1 / 2 \\
4 x^{2} \log \left(|x|^{-1}\right)+2 x^{2} & \text { if } \beta=-1 / 2 .\end{cases}
\end{array}
$$

Inserting (4.8) for $\beta<-1 / 2$ we obtain

$$
\begin{aligned}
\int_{A_{11}} & \min \left(1,|x g(s)|^{2}\right) \nu(\mathrm{d} x) \mathrm{d} s \\
\quad= & \int_{|x| \leq 1}\left(\frac{2 x^{2}}{2 \beta+1}\left(1-|x|^{-\frac{2 \beta+1}{\beta}}\right)+2|x|^{-1 / \beta}\right) \nu(\mathrm{d} x)<\infty
\end{aligned}
$$

where the inequality follows by an application of (b). A similar application of (4.8) shows that (4.9) is finite for $\beta=-1 / 2$ using (c). For $\beta>-1 / 2$ we have

$$
\int_{A_{11}} \min \left(1,|x g(s)|^{2}\right) \mathrm{d} s \nu(\mathrm{d} x) \leq\left(\int_{|x| \leq 1} x^{2} \nu(\mathrm{d} x)\right)\left(\int_{|s| \leq 1}|s|^{2 \beta} \mathrm{d} s\right)<\infty .
$$

Since $A_{12}=\{(s, x) \in \mathbb{R} \times \mathbb{R}:|s| \leq 1,|x|>1\}$ we have

$$
\begin{aligned}
\int_{A_{12}} \min \left(1,|x g(s)|^{2}\right) \mathrm{d} s \nu(\mathrm{d} x) & \leq \int_{\{|x|>1\}} \int_{\{|s| \leq 1\}} 1 \mathrm{~d} s \nu(\mathrm{d} x) \\
& \leq 2 \nu(x \in \mathbb{R}:|x|>1)<\infty
\end{aligned}
$$

since $\nu$ is a Lévy measure. For the last area, $A_{21}$, we have

$$
\int_{A_{21}} \min \left(1,|x g(s)|^{2}\right) \mathrm{d} s \nu(\mathrm{d} x)=2\left(\int_{|x| \leq 1} x^{2} \nu(\mathrm{d} x)\right)\left(\int_{1}^{\infty} s^{-2 \gamma} \mathrm{d} s\right)<\infty
$$

since $\nu$ is a Lévy measure and $\gamma>1 / 2(c f$. (a)). Hence, the integral (4.5) is finite and the proof is complete.

Proof of Theorem 4.1. For all $s \in \mathbb{R} \backslash\{0\}$ let

$$
g(s)=\left(a\left(s_{+}\right)^{-H-1 / \alpha}+b\left(s_{-}\right)^{-H-1 / \alpha}\right) \operatorname{sign}(s)
$$


where $\operatorname{sign}(s):=\mathbb{1}_{(0, \infty)}(s)-\mathbb{1}_{(-\infty, 0)}(s)$ denotes the sign function, and $g(0)=0$. For a fixed $t \in \mathbb{R}$ let $f: \mathbb{R} \rightarrow \mathbb{C}$ denote the integrand of the harmonizable fractional Lévy motion, that is,

$$
f(s)=i\left(1-e^{i t s}\right) g(s), \quad s \in \mathbb{R} .
$$

We want to apply Lemma 4.2 on $|f|: \mathbb{R} \rightarrow \mathbb{R}_{+}$and the Lévy measure $\nu \circ(x \mapsto|x|)^{-1}$ on $\mathbb{R}$. Observe that $|f|$ is bounded on $[-1,1]^{c}$, and

$$
|f(s)|=O\left(|s|^{1-H-1 / \alpha}\right) \text { as } s \rightarrow 0, \quad \text { and } \quad|f(s)|=O\left(|s|^{-H-1 / \alpha}\right) \text { as }|s| \rightarrow \infty .
$$

Hence, with $\beta:=1-H-1 / \alpha \in \mathbb{R}$ and $\gamma=H+1 / \alpha \in(0, \infty),|f|$ satisfies condition (4.2). Condition (a) of Lemma 4.2 reads $H+1 / \alpha>1 / 2$ (which follows since $\alpha<2$ and $H>0$ ) and

$$
\int_{|x|>1}|x|^{\frac{1}{H+1 / \alpha}} \nu(\mathrm{d} x)<\infty
$$

which follows by assumption (a) of Theorem 4.1. Note that $\beta<-1 / 2$ if and only if $H+1 / \alpha>3 / 2$, and therefore condition (b) of Lemma 4.2 reads

$$
\int_{|x| \leq 1}|x|^{\frac{1}{H+1 / \alpha-1}} \nu(\mathrm{d} x)<\infty \quad \text { whenever } \quad H+1 / \alpha>3 / 2,
$$

which follows by assumption (b) of Theorem 4.1. Since $\beta=-1 / 2$ corresponds to $H+1 / \alpha=3 / 2$, condition (c) of Lemma 4.2 follows by condition (c) of Theorem 4.1. According to Lemma 4.2,

$$
\int_{\mathbb{R}} \int_{\mathbb{R}^{2}} \min \left(1,|x f(s)|^{2}\right) \nu(\mathrm{d} x) \mathrm{d} s<\infty
$$

which shows that the stochastic integral $\int f \mathrm{~d} L$ exists, $c f$. Theorem 3.1, and hence $X_{t}$ is well-defined for all $t \in \mathbb{R}$.

The stationary increments of $X$ follows from an application of (3.3). To show that $X$ is isotropic it is enough to show that $Z=\int \psi \mathrm{d} L$ is isotropic for all $L$-integrable functions $\psi: \mathbb{R} \rightarrow \mathbb{C}$. For any $2 \times 2$ matrix $A, \varphi_{A \tilde{Z}}(\theta)=$ $\varphi_{\tilde{Z}}\left(A^{T} \theta\right)$, which in conjunction with (3.3) implies that $Z$ is isotropic. Since $f$ satisfies $|f(s)|=2|\sin (t s / 2) g(s)|$, (4.1) follows also from (3.3).

We are now ready to complete the proof of Theorem 1.3.

Proof of Theorem 1.3. Throughout the proof $c$ will denote a finite constant which may change from line to line. We will start by showing that process $X$ is well-defined. For any function $\psi: \mathbb{R}_{+} \rightarrow \mathbb{R}_{+}$we have by Assumption (A) that

$$
\begin{aligned}
& \int_{\mathbb{R}^{2}} \psi(|x|) \nu(\mathrm{d} x)=\int_{\mathbb{R}^{2}} \psi(|x|) h(x) \mathrm{d} x \\
& \quad \leq C \int_{\mathbb{R}^{2}} \psi(|x|)|x|^{-2-\alpha} \mathrm{d} x=2 \pi C \int_{0}^{\infty} \psi(u) u^{-1-\alpha} \mathrm{d} u
\end{aligned}
$$

where the last equality follows from the change to polar coordinates. In particular,

$$
\int_{|x|>1}|x|^{\frac{1}{H+1 / \alpha}} \nu(\mathrm{d} x) \leq c \int_{1}^{\infty} v^{\frac{1}{H+1 / \alpha}} v^{-\alpha-1} \mathrm{~d} v<\infty
$$


where the last inequality follows since $H>0$. By (4.12), condition (a) of Theorem 4.1 is satisfied. To show (b) of Theorem 4.1 we assume that $H+1 / \alpha>3 / 2$. By (4.11) we have that

$$
\int_{|x| \leq 1}|x|^{\frac{1}{H+1 / \alpha-1}} \nu(\mathrm{d} x) \leq c \int_{0}^{1} v^{\frac{1}{H+1 / \alpha-1}} v^{-\alpha-1} \mathrm{~d} v<\infty
$$

where the last inequality follows since $H<1$ and $H+1 / \alpha-1>0$ (which is implied by $H+1 / \alpha>3 / 2$ ). This shows that condition (b) of Theorem 4.1 holds. Furthermore,

$$
\int_{|x| \leq 1}|x|^{2} \log \left(|x|^{-1}\right) \nu(\mathrm{d} x) \leq c \int_{0}^{1} v^{2} \log \left(v^{-1}\right) v^{-1-\alpha} \mathrm{d} v<\infty
$$

since $\alpha<2$, which shows that condition (c) of Theorem 4.1 holds. Thus, process $X$ exists, has stationary increments and an isotropic distribution, $c f$. Theorem 4.1.

In the following we will study the characteristic function of the finite dimensional distributions for the lefthand side of (1.7) and show convergence towards the characteristic function of harmonizable fractional stable motion. For $n \in \mathbb{N}, t_{1}, \ldots, t_{n} \in \mathbb{R}, a_{1}, \ldots, a_{n} \in \mathbb{C}$ and $\epsilon>0$ set

$$
Z_{\epsilon}=\epsilon^{-H} \sum_{j=1}^{n} a_{j} X_{\epsilon t_{j}} \quad \text { and } \quad f_{\epsilon}(s)=\epsilon^{-H} \sum_{j=1}^{n} a_{j}\left(1-e^{i t_{j} \epsilon s}\right) i g(s)
$$

for all $s \in \mathbb{R}$, where $g$ is given by (4.10). By (3.3) we have that

$$
\log \varphi_{Z_{\epsilon}}(\theta)=\int_{\mathbb{R}} K\left(\left|f_{\epsilon}(s)\right| \theta\right) \mathrm{d} s,
$$

and since

$$
f_{\epsilon}\left(\epsilon^{-1} s\right)=f_{1}(s) \epsilon^{1 / \alpha} \quad \text { for all } \epsilon>0, s \in \mathbb{R},
$$

the substitution $u=\epsilon s$ yields

$$
\log \varphi_{Z_{\epsilon}}(\theta)=\epsilon^{-1} \int_{\mathbb{R}} K\left(\left|f_{1}(u)\right| \epsilon^{1 / \alpha} \theta\right) \mathrm{d} u .
$$

For $u \in \mathbb{R}^{2}$, the substitution $v=\epsilon^{1 / \alpha} x$ shows that

$$
\begin{aligned}
K\left(u \epsilon^{1 / \alpha}\right) & =\int_{\mathbb{R}^{2}}\left(\cos \left(\left\langle u \epsilon^{1 / \alpha}, x\right\rangle\right)-1\right) h(x) \mathrm{d} x \\
& =\epsilon^{-2 / \alpha} \int_{\mathbb{R}^{2}}(\cos (\langle u, v\rangle)-1) h\left(\epsilon^{-1 / \alpha} v\right) \mathrm{d} v
\end{aligned}
$$

By assumption (1.5),

$$
\frac{h\left(\epsilon^{-1 / \alpha} v\right)}{\epsilon^{1+2 / \alpha}} \rightarrow c_{0}|v|^{-2-\alpha} \quad \text { as } \epsilon \rightarrow 0_{+}
$$


for all $v \in \mathbb{R}^{2} \backslash\{0\}$. Due to assumption (1.6) we have

$$
\left|\frac{h\left(\epsilon^{-1 / \alpha} v\right)}{\epsilon^{1+2 / \alpha}}\right| \leq C|v|^{-2-\alpha}, \quad \text { for all } v \in \mathbb{R}^{2} \backslash\{0\}, \epsilon>0 .
$$

For all $y \in \mathbb{R}^{2}$,

$$
\int_{\mathbb{R}^{2}} \frac{1-\cos (\langle y, v\rangle)}{|v|^{2+\alpha}} \mathrm{d} v=c_{1}|y|^{\alpha}<\infty
$$

where the constant $c_{1}$ is explicitly given in Exercise 18.9 of [16]. Moreover, $f_{1} \in L^{\alpha}(\mathbb{R})$ due the existence of the harmonizable fractional stable motion. Hence, by Lebesgue's dominated convergence theorem and (4.13), (4.14) and (4.15) we deduce that

$$
\log \varphi_{Z_{\epsilon}}(\theta) \rightarrow-c_{0} c_{1} \int_{\mathbb{R}}\left|f_{1}(u) \theta\right|^{\alpha} \mathrm{d} u \quad \text { as } \epsilon \rightarrow 0_{+} .
$$

By the continuity theorem for characteristic functions, the convergence in distribution (1.7) follows by (4.16), and the proof is complete.

Acknowledgements. A. Basse-O'Connor and T. Grønbæk's research were supported by the grant DFF-4002-00003 from the Danish Council for Independent Research. M. Podolskij gratefully acknowledges financial support through the research project "Ambit fields: probabilistic properties and statistical inference" funded by Villum Fonden. Finally, we would like to thank an anonymous referee for very detailed and constructive remarks and corrections.

\section{REFERENCES}

[1] J.-M. Bardet and D. Surgailis, Nonparametric estimation of the local Hurst function of multifractional Gaussian processes. Stochastic Process. Appl. 123 (2013) 1004-1045.

[2] A. Basse-O'Connor, R. Lachièze-Rey and M. Podolskij, Power variation for a class of stationary increments Lévy driven moving averages. Ann. Probab. 45 (2017) 4477-4528.

[3] A. Benassi, S. Cohen and J. Istas, Identification and properties of real harmonizable fractional Lévy motions. Bernoulli 8 (2002) 97-115.

[4] A. Benassi, S. Cohen and J. Istas, On roughness indices for fractional fields. Bernoulli 10 (2004) 357-373.

[5] A. Benassi, S. Jaffard and D. Roux, Elliptic Gaussian random processes. Rev. Mat. Iberoamericana 13 (1997) 19-90.

[6] S. Cambanis and M. Maejima, Two classes of self-similar stable processes with stationary increments. Stochastic Process. Appl. 32 (1989) 305-329.

[7] J.L. Doob, Vol. 7 of Stochastic Processes. Wiley, New York (1953).

[8] D. Kremer and H.-P. Scheffler, Multivariate stochastic integrals with respect to independently scattered random measures on $\delta$-rings. Preprint arXiv:1711.00890 [math.PR] (2018) 28.

[9] J. Lamperti, Semi-stable stochastic processes. Trans. Am. Math. Soc. 104 (1962) 62-78.

[10] T. Marquardt, Fractional Lévy processes with an application to long memory moving average processes. Bernoulli 12 (2006) 1099-1126.

[11] R.-F. Peltier and J. Lévy Véhel, Multifractional Brownian Motion: Definition and Preliminary Results. Research Report RR-2645, INRIA (1995). Available at https://hal.inria.fr/inria-00074045/file/RR-2645.pdf.

[12] B.S. Rajput and J. Rosiński, Spectral representations of infinitely divisible processes. Probab. Theory Related Fields 82 (1989) 451-487.

[13] J. Rosiński, On the structure of stationary stable processes. Ann. Probab. 23 (1995) 1163-1187.

[14] J. Rosiński and G. Samorodnitsky, Classes of mixing stable processes. Bernoulli 2 (1996) 365-377.

[15] G. Samorodnitsky and M.S. Taqqu, Stable non-Gaussian Random Processes. Stochastic Modeling. Chapman \& Hall, New York (1994). Stochastic models with infinite variance.

[16] K. Sato, Lévy processes and infinitely divisible distributions. Vol. 68 of Cambridge Studies in Advanced Mathematics. Cambridge University Press, Cambridge (1999). Translated from the 1990 Japanese original, Revised by the author.

[17] S. Stoev, M.S. Taqqu, C. Park, G. Michailidis and J. Marron, Lass: a tool for the local analysis of self-similarity. Comput. Stat. Data Anal. 50 (2006) 2447-2471.

[18] K. Urbanik, Random measures and harmonizable sequences. Stud. Math. 31 (1968) 61-88. 
[19] W. Willinger, M.S. Taqqu and A. Erramilli, A bibliographical guide to self-similar traffic and performance modeling for modern high-speed networks. Stochastics Networks: Theory and Applications. Oxford University Press (1996) 339-366.

[20] A.M. Yaglom, An Introduction to the Theory of Stationary Random Functions. Courier Corporation (2004). 